\author{
Research Article \\ www.ijrap.net (ISSN:2229-3566)
}

\title{
EVALUATION OF EFFECT OF UDVARTANA ON MEDO DHATU USING BODY COMPOSITION MONITOR
}

\author{
Jaseena C. ${ }^{1}$, Annie Yohannan ${ }^{2}$, Anjali Sivaram ${ }^{3}$, Anjana R. *4 \\ ${ }^{1}$ Associate Professor, Department of Kriya Shareera, KMCT Ayurveda Medical College, Manassery, Kozhikode, \\ Kerala, India \\ ${ }^{2}$ Principal, Government Ayurveda College, Department of Kriya Shareera, Pariyaram, Kannur, Kerala, India \\ ${ }^{3}$ Associate Professor, Department of Kriya Shareera, Government Ayurveda College, Pariyaram, Kannur, Kerala, India \\ ${ }^{4}$ Assistant Professor, Department of Kaumarabhritya, KMCT Ayurveda Medical College, Manassery, Kozhikode, \\ Kerala, India
}

Received on: 10/04/20 Accepted on: 11/05/20

\begin{abstract}
*Corresponding author
E-mail: anjanachintu@gmail.com
\end{abstract}

DOI: $10.7897 / 2277-4343.110490$

\begin{abstract}
The aim of the study was to evaluate the effect of udvartana on medo dhatu using body composition monitor. The study samples were 30 normal healthy individuals with BMI between 25-29.9 and 30-40 years in age. Degree of vitiation of medo dhatu was assessed using a detailed proforma. Their routine blood parameters and lipid profile were assessed. Body weight, BMI, body fat percentage, skeletal muscle percentage, visceral fat etc. was analyzed using body composition monitor. Udvartana with triphala choorna was performed for 30 minutes for 7 days. Reassessment of parameters was done after 7 days and 14 days. The procedure showed significant reduction in the degree of vitiation of medo dhatu. The parameters such as total cholesterol, VLDL, LDL, TG showed significant reduction with enhancement of HDL. Body weight, BMI, body fat percentage, skeletal muscle percentage and visceral fat levels were also reduced significantly after the udvartana.
\end{abstract}

Keywords: Udvartana, medo dhatu, body composition monitor, Triphala choorna, BMI, lipid profile

\section{INTRODUCTION}

Obesity is becoming a serious health issue in both the developed and developing countries. ${ }^{1}$ As per British Medical Journal onethird of the population worldwide are suffering from overweight and obesity. ${ }^{2}$ They also reported that 30,000 deaths occur per year due to obesity. ${ }^{3}$ According to WHO reports-2008, obesity is included in one among the 10 selected health risks. ${ }^{4}$ There are about 300 billions of people worldwide clinically living with obesity. ${ }^{5}$ A study conducted in adult population of urban Delhi and rural Ballabgarh (Haryana State), revealed that overweight was widely prevalent in the urban population (men: $35.1 \%$, women: $47.6 \%$ ) compared to the rural population (men: $7.7 \%$, women: $11.3 \%) .{ }^{6}$ Universal incidence of mortality and morbidity is also highly alarming due to the secondary complication of the obesity. $^{7}$

Udvartana, one among the daily regimens for the maintenance of Swasthya, will be helpful to eliminate the doshas aggravated by faulty lifestyles. Acharyas had mentioned that udvartana is effective for the management of sthaulya. It is having properties like kapha hara, medovilayana, angasthirikarana etc. Moreover, udvartana is a simple process and has no harmful effect when compared with other fat reducing packages and treatments offered by present era. Many studies have been conducted to manage sthaulya using oral medicines. It is the nature of human to hate oral medication than other routes due to its distaste, belching etc. Triphala, one of the popular combinations in Ayurveda pacifies kaphapitta doshas. Udvartana performed with triphala choorna in the management of overweight is anticipated to make a significant result.

\section{MATERIALS AND METHODS}

\section{Objective}

To study the effect of udvartana on medo dhatu using body composition monitor.

\section{Source of data}

For obtaining the samples, an advertisement was given in the daily newspapers. The data were collected from individuals who attended outpatient department of Kriya Shareera, Government Ayurveda College Hospital, Kannur district, Kerala state in response to advertisement.

\section{Ethical clearance}

Ethical clearance was obtained from Institutional Ethics Committee of Government Ayurveda College, Kannur district, Kerala state (E2-3971/11-ACK).

\section{Inclusion criteria}

Normal healthy individuals of both gender between the age group of 30 to 40 years having BMI between 25 and 29.9

\section{Exclusion criteria}

Subjects with systemic and mental diseases, hyperlipidaemia and under any sort of medication, pregnant and lactating women 


\section{Sampling technique}

Simple random sampling

\section{Type of study}

Intervention

\section{Period of study}

6 months

\section{Sample size}

30

\section{Materials}

To check body fat percentage, skeletal muscle percentage, BMI etc. body composition monitor was used. The drug which was used for the procedure of udvartana, i.e., triphala choorna was purchased from Aushadhi, Pariyaram, Kerala state (Pin-670142). For collection of blood sample needle, disposable syringe, tourniquet, spirit, cotton and EDTA vial were used. For assessing lipid profile semi auto analyser (Figure 1) was used. A proforma was used for the assessment of vitiation of medo dhatu.

\section{Body Composition Monitor}

\section{Principle}

This instrument estimates the body fat percentage by the Bio electrical impedance method. Muscles or blood vessels are body tissues with a high water content that conducts electricity easily. Body fat is the tissue that has low electric conductivity. This instrument sends an extremely low electrical current of $50 \mathrm{kHz}$ and less than $500 \mu \mathrm{A}$ through the body to determine the amount of fat tissue. This weak electrical current is not felt while operating this instrument. This is an instrument suitable to measure the body fat percentage, skeletal muscle percentage, visceral fat level, body weight and BMI (Figures 2 and 3). ${ }^{8}$

\section{Body weight}

Maintaining an ideal weight can help to prevent obesity or weight loss and other diseases and lead a longer life. Ideal weight $(\mathrm{kg})=$ $22 \times$ height $(\mathrm{m}) \mathrm{x}$ height $(\mathrm{m})$. This may not be applicable for professional athletes and body builders etc, with higher ratios of muscular development.

\section{Body Mass Index (BMI or Quetelet's Index)}

The BMI is the actual body weight divided by the height square $\left(\mathrm{Kg} / \mathrm{m}^{2}\right)$. This index more closely corresponds to measurements of body fat and better differentiates over weight due to an increase in muscle mass from true obesity; devised by the Belgian polymath Adolphe Quetelet. The international classification of BMI which is widely accepted is as follows:

- Under weight $-<18.5 \mathrm{~kg} / \mathrm{m}^{2}$

- Normal weight $-18.5-24.9 \mathrm{~kg} / \mathrm{m}^{2}$

- Overweight - $25-29.9 \mathrm{Kg} / \mathrm{m}^{2}$

- Obesity (class - I) $30-34.9 \mathrm{Kg} / \mathrm{m}^{2}$

- Obesity (class - II) $35-39.9 \mathrm{Kg} / \mathrm{m}^{2}$

- Severe or morbid obesity (class - III) $>40 \mathrm{~kg} / \mathrm{m}^{2}$

- Ideal BMI is $22^{9}$

\section{Body Fat Percentage}

Body fat is classified as subcutaneous fat and visceral fat etc, depending on where it is located in the body. Body fat percentage refers to the amount of body fat mass in regards to the total body weight expressed as a percentage.

Body fat percentage $(\%)=\{$ body fat mass $(\mathrm{kg}) /$ body weight $(\mathrm{kg})\} \times 100$

Body fat percentage serves a vital role in storing energy, protecting internal organs, etc. Too much and too little body fat is unhealthy. The distribution of body fat in males and females is different (Table 1). ${ }^{10}$

\section{Visceral Fat Level}

Visceral fat is the fat surrounding internal organs. Too much visceral fat is thought to be closely linked increased levels of fat in the blood stream, which can lead to common diseases such as hyperlipidemia and diabetes. In order to prevent or improve conditions of common diseases, it is important to try and reduce the visceral fat levels to an acceptable level. People with high visceral fat levels tend to have large stomach. ${ }^{11}$

\section{Skeletal Muscle}

Muscle is divided in to two types; muscle in internal organs, such as the heart and muscle attached to bones that are used to move the body. The skeletal muscle percentage in average female is approximately $28 \%$ and that in average males is $37 \%$.

\section{Triphala Choorna}

In order to maintain the quality, the whole amount of triphala choorna was purchased from the same company i.e. Aushadhi, Pariyaram with the same batch number. The powder was of $80 /+100$ mesh sieve size. ${ }^{12}$

\section{Rationale for Selecting Triphala}

- According to Acharya Sushruta, triphala is kushta hara. It does not produce any harmful effect to the skin. So this drug was selected.

- The drugs of triphala possess laghu and ruksha properties. Acharya Sushruta has described the lekhana property of laghu guna. In this study, the selected individuals are overweight people having BMI 25-29.9. So the laghu and ruksha properties of triphala would act on the kapha and Meda of the individuals.

\section{Blood Investigations}

Relevant blood investigations i.e. routine blood examination and lipid profile had been performed.

\section{Assessment of routine blood examination}

To maintain the accuracy and standardization of the results, the routine blood examinations were carried out at clinical laboratory of Govt. Ayurveda College Hospital, Kannur.

\section{Assessment of serum content}

All the investigations were done with the help of semi auto analyser using the biochemical reagents kits available in the market. Kits of the same company and of same batch numbers were sustained for each and every investigation throughout this study. 


\section{Proforma}

The proforma consisted of:

- First part: consisted of preliminary data, data regarding personal history.

- Second part: consisted of vital data, systemic examinations and Ayurvediya Dashvidha Pariksha and assessment parameters.

The demographic data of each individual were collected based on this proforma. In this proforma the normal and vitiated levels of medo dhatu in individuals had been graded. Grading of medo dhatu in the proforma is purely based on descriptions in Ayurvedic classics including subjective and some objective characteristics.

Criteria to assess medo dhatu

A. Subjective criteria

B. Objective criteria

\section{Subjective criteria}

This includes certain subjective characters shown by medo dhatu in a person in normalcy and vitiated states, either increase or decrease, as explained in Ayurvedic classics. Each character shown in normalcy had been given Grade 1. While in vitiated states hold higher grading 2 and 3 depending on the degree of vitiation. For example if the eyes are "normal unctuous" the grade will be 1 , if the eyes having "minimal secretions or slightly increased secretion" the grade will be 2 and if the eyes having "rarely secretions or more secretions" the grade will be 3 .

\section{Objective criteria}

This includes certain objective characteristics shown by medo dhatu in normalcy, and vitiated condition as mentioned in Ayurvedic classics. Here also grading had been done in the same manner as that of subjective characters. For example in the case of body built, if it is "stable" the score will be 1 , if it is "overweight or emaciated" the score will be 2, and if it is "obese or increased emaciation" the score will be 3 . Body built was assessed on the basis of BMI. Like this, the grading of 22 characteristics of medo dhatu was performed. In the end, the sum of the grading of all these 22 characteristics had been calculated to get a total score, which showed the state of medo dhatu. Score 22 shows normal state of medo dhatu and above 22 shows the vitiated state of medo dhatu.

\section{Methods}

Udvartana was done for 30 minutes for 7 days. Using body composition monitor, the body weight, BMI, body fat percentage, skeletal muscle percentage and visceral fat level of all the participants were recorded before and after the intervention. Their blood parameters like $\mathrm{Hb}$, TLC, DLC, ESR and lipid profile were also evaluated before and after the intervention. The state of medo dhatu was also noted before and after the intervention.

\section{Udvartana}

Udvartana is massage technique of whole body below the neck with powders of medicinal plants in pratiloma direction of hairs in the body.

\section{Materials Used}

For the procedure

- Droni (Abhyanga table): For doing the procedure

- Triphala choorna for doing udvartana: Triphala choorna of $80 /+100$ mesh sieve size was used.

- Heater and vessel: For heating of powder.

The subjects were advised to take bath after one hour of the procedure without using soap. The masseurs were advised to wear apron and mask while doing udvartana.

\section{Preparation of the Subject}

\section{A) Preliminary procedures}

1. Confirm that the subject is fit for the procedure.

2. Each subject was given a brief description about the procedure.

3. Ensure that the subject has evacuated bowels and voided urine so that he / she will not have such urges during the procedure.

4. Ensure that all the equipments and materials are ready.

\section{B) Massage}

1. Verify and satisfy that everything is okay.

2. Check the vitals.

3. Powder should be reasonably hot.

\section{Procedure}

A handful of choorna was taken and rubbed it over the patient's body in pratiloma direction.

\section{Position of Udvartana}

Subject in minimum dress is made to lie down on the Droni (Abhyanga table). Udvartana is carried out in 7 different positions of the subject. The seven postures are: sitting, supine, right lateral, supine, left lateral, supine and sitting. Every part of the body below the neck is subjected to massaging one after the other exerting firm pressure.

\section{Procedure of Data Collection}

Thirty healthy individuals both males and females in and around Government Ayurveda College, Kannur, of age group 30-40 years, having BMI between 25-29.9 were included in the study. They were asked about their diet. To avoid the possibility of taking extra calories, a diet chart sticking to his/ her normal diet was prepared and given to each subject. The subjects were advised to take light food before udvartana. In the beginning day of research work $3 \mathrm{ml}$ of venous blood was taken for before trial serum investigations. Thus, extracted samples of blood were labelled carefully by mentioning subject's name, sample number, whether before trial or after trial sample and the time of collection. The blood required for routine examination was taken and the remaining sample was allowed to clot at room temperature for half an hour for serum investigations. These samples for serum investigations are then centrifuged for 15-20 minutes with a speed of $2500 \mathrm{rpm}$. Before subjecting the individual to udvartana, their body weight, BMI, body fat percentage, skeletal muscle percentage, visceral fat level were recorded. The vitals also had been recorded. The udvartana was done between 7 am and 9 am, to exclude the diurnal variations. Similarly to rule out seasonal variations the entire study was 
conducted within a period of 2 months. The selected individuals were subjected to 30 minutes udvartana for 7 days.

\section{Assessment Parameters of Vitality}

In this present study, daily monitoring of the following vitals had been performed before and after the udvartana procedure.

\section{Blood pressure}

Using a sphygmomanometer and stethoscope, blood pressure of all the subjects were recorded.

\section{Pulse rate}

The individual was asked to sit comfortably in front of the examiner and allowed to feel relaxed and then the pulse of the radial artery at left wrist joint was counted for one minute by using the index, middle and ring fingers of right hand of the examiner and a stop watch.

\section{Temperature}

Individual's body temperature was recorded by using the standard clinical thermometer.

\section{Monitoring of study subjects}

After 7 days of udvartana, blood investigations, the body parameters and the state of medo dhatu were assessed. Follow up assessment of all the subject was done 1 week after the procedure, which include blood investigations, the assessment of body parameters and the state of medo dhatu.

\section{Data analysis}

The data was entered in to the master sheet and various statistical tables were constructed. The statistical constants like mean, standard deviation (SD) and percentage were computed. The effect of udvartana on body parameters, vital data, blood investigations and medo dhatu were statistically analysed and determined by using the 2 tailed $t$ tests.

Table 1: Interpreting the body fat percentage result

\begin{tabular}{|c|c|c|c|c|}
\hline Gender & - (Low) & 0 (Normal) & + (High) & ++ (very high) \\
\hline Female & $5.5 .0-19.9 \%$ & $20.20-29.9 \%$ & $30.0-34.9 \%$ & $35.0-50.0 \%$ \\
\hline Male & $5.0-9.9 \%$ & $10.0-19.9 \%$ & $20.0-24.9 \%$ & $25.0-50 \%$ \\
\hline
\end{tabular}

Table 2: Effect on body weight

\begin{tabular}{|c|c|c|c|c|c|c|}
\hline Body Weight & Mean & $\mathbf{N}$ & SD & SE & T value & P value \\
\hline $\begin{array}{l}\text { Pre } \\
\text { Post }\end{array}$ & $\begin{array}{l}73.7780 \\
72.9570\end{array}$ & 30 & $\begin{array}{l}10.27545 \\
10.17951\end{array}$ & $\begin{array}{l}1.87603 \\
1.85851\end{array}$ & 3.839 & 0.001 \\
\hline $\begin{array}{c}\text { Post } \\
\text { Follow up } \\
\end{array}$ & $\begin{array}{l}72.9570 \\
73.3510 \\
\end{array}$ & 30 & $\begin{array}{l}10.17951 \\
10.36050 \\
\end{array}$ & $\begin{array}{l}1.85851 \\
1.89156 \\
\end{array}$ & 1.654 & 0.109 \\
\hline $\begin{array}{c}\text { Pre } \\
\text { Follow up }\end{array}$ & $\begin{array}{l}73.7780 \\
73.3510\end{array}$ & 30 & $\begin{array}{l}10.27545 \\
10.36050\end{array}$ & $\begin{array}{l}1.87603 \\
1.89156\end{array}$ & 2.819 & 0.009 \\
\hline
\end{tabular}

Table 3: Effect on BMI

\begin{tabular}{|c|c|c|c|c|c|c|}
\hline BMI & Mean & N & SD & SE & T value & P value \\
\hline Pre & 27.143 & \multirow{2}{*}{30} & 1.45500 & 0.26565 & 2.542 & 0.017 \\
Post & 26.9133 & & 1.51310 & 0.27625 & & \\
\hline Post & 26.9133 & \multirow{2}{*}{30} & 1.51310 & 0.27625 & -1.233 & 0.227 \\
Follow up & 27.0167 & & 1.47603 & 0.26949 & & \\
\hline Pre & 27.143 & \multirow{2}{*}{30} & 1.45500 & 0.26565 & 2.096 & 0.045 \\
Follow up & 27.0167 & & & & \\
\hline \multicolumn{7}{|c|}{ SD - standard deviation; SE - standard error; significant when $\mathrm{p} \leq 0.05$} \\
\hline
\end{tabular}

Table 4: Effect on body fat percentage

\begin{tabular}{|c|c|c|c|c|c|c|}
\hline Body Fat Percentage & Mean & N & SD & SE & T value & P value \\
\hline Pre & 37.5667 & \multirow{2}{*}{30} & 5.83363 & 1.06507 & 3.521 & 0.001 \\
Post & 37.0200 & & 5.84940 & 1.06795 & & \\
\hline Post & 37.0200 & 30 & 5.84940 & 1.06795 & -.946 & 0.352 \\
Follow up & 37.2100 & & 5.66473 & 1.03423 & & \\
\hline Pre & 37.5667 & 30 & 5.83363 & 1.06507 & 2.763 & 0.010 \\
Follow up & 37.2100 & & & & \\
\hline \multicolumn{2}{|c|}{ SD - standard deviation; SE - standard error; significant when $\mathrm{p} \leq 0.05$} \\
\hline
\end{tabular}


Jaseena C. et al / Int. J. Res. Ayurveda Pharm. 11 (4), 2020

Table 5: Effect on skeletal muscle percentage

\begin{tabular}{|c|c|c|c|c|c|c|}
\hline Skeletal muscle percentage & Mean & $\mathbf{N}$ & SD & SE & T value & P value \\
\hline Pre & 25.05 & \multirow{2}{*}{30} & 4.06191 & 0.74160 & \multirow[t]{2}{*}{2.796} & \multirow[t]{2}{*}{0.009} \\
\hline Post & 24.83 & & 4.08750 & 0.74627 & & \\
\hline Post & 24.83 & \multirow{2}{*}{30} & 4.0875 & 0.74627 & \multirow[t]{2}{*}{2.041} & \multirow[t]{2}{*}{0.050} \\
\hline Follow up & 24.07 & & 4.20797 & 0.76827 & & \\
\hline Pre & 25.05 & \multirow{2}{*}{30} & 4.06191 & 0.74160 & \multirow[t]{2}{*}{2.563} & \multirow[t]{2}{*}{0.016} \\
\hline Follow up & 24.07 & & 4.20797 & 0.76827 & & \\
\hline
\end{tabular}

SD - standard deviation; SE - standard error; significant when $\mathrm{p} \leq 0.05$

Table 6: Effect on visceral fat level

\begin{tabular}{|c|c|c|c|c|c|c|}
\hline Visceral Fat Level & Mean & $\mathbf{N}$ & SD & SE & T value & P value \\
\hline Pre & 14.733 & 30 & 4.98918 & 0.91090 & 3.071 & 0.005 \\
\hline Post & 14.433 & & 4.96667 & 0.90679 & & \\
\hline Post & 14.433 & 30 & 4.96667 & 0.90679 & -1.072 & 0.293 \\
\hline Follow up & 14.5667 & & 4.96667 & 0.90679 & & \\
\hline Pre & & 30 & 4.98918 & & 1.542 & 0.134 \\
\hline Follow up & 14.5667 & & 4.96667 & 0.90679 & & \\
\hline
\end{tabular}

$\mathrm{SD}$ - standard deviation; SE - standard error; significant when $\mathrm{p} \leq 0.05$

Table 7: Effect on haemoglobin in males

\begin{tabular}{|c|c|c|c|c|c|c|}
\hline Hb (males) & Mean & n & SD & SE & T value & P value \\
\hline $\begin{array}{c}\text { Pre } \\
\text { Post }\end{array}$ & 12.93 & \multirow{2}{*}{30} & 1.033 & 0.298 & 2.69 & 0.012 \\
\hline Post & 13.28 & & 1.008 & 0.291 & & \\
Follow up & 13.28 & 30 & 1.008 & 0.291 & -0.895 & 0.390 \\
\hline Pre & 12.93 & \multirow{2}{*}{30} & 1.033 & 0.298 & -0.917 & 0.379 \\
Follow up & 13.13 & & 1.026 & 0.296 & & \\
\hline
\end{tabular}

$\mathrm{SD}$ - standard deviation; SE - standard error; significant when $\mathrm{p} \leq 0.05$

Table 8: Effect on haemoglobin in females

\begin{tabular}{|c|c|c|c|c|c|c|}
\hline Hb (females) & Mean & n & SD & SE & T value & P value \\
\hline Pre & 12.45 & \multirow{2}{*}{30} & 1.125 & 0.265 & -1.750 & 0.098 \\
Post & 12.7 & & 1.105 & 0.260 & & \\
\hline Post & 12.7 & \multirow{2}{*}{30} & 1.105 & 0.260 & 0.511 & 0.616 \\
Follow up & 12.59 & & 1.234 & 0.291 & & \\
\hline Pre & 12.45 & \multirow{2}{*}{30} & 1.125 & 0.265 & -1.250 & 0.228 \\
Follow up & 12.59 & & 1.234 & 0.291 & & \\
\hline
\end{tabular}

SD - standard deviation; SE - standard error; significant when $\mathrm{p} \leq 0.05$

Table 9: Effect on TLC

\begin{tabular}{|c|c|c|c|c|c|c|}
\hline TC & Mean & n & SD & SE & T value & P value \\
\hline Pre & $8.2733 \times 10^{3}$ & & $1.69032 \times 10^{3}$ & $0.30861 \times 10^{3}$ & 3.260 & 0.003 \\
Post & $7.7167 \times 10^{3}$ & 30 & & $1.63371 \times 10^{3}$ & $0.29827 \times 10^{3}$ & \\
\hline Post & $7.7167 \times 10^{3}$ & & $1.63371 \times 10^{3}$ & $0.29827 \times 10^{3}$ & -1.151 & 0.259 \\
Follow up & $7.8867 \times 10^{3}$ & 30 & & $1.54601 \times 10^{3}$ & $0.28226 \times 10^{3}$ & \\
\hline Pre & $8.2733 \times 10^{3}$ & \multirow{2}{*}{30} & $1.69032 \times 10^{3}$ & $0.30861 \times 10^{3}$ & 2.38 & 0.024 \\
Follow up & $7.8867 \times 10^{3}$ & & $1.54601 \times 10^{3}$ & $0.28226 \times 10^{3}$ & & \\
\hline
\end{tabular}

SD - standard deviation; SE - standard error; significant when $\mathrm{p} \leq 0.05$ 
Jaseena C. et al / Int. J. Res. Ayurveda Pharm. 11 (4), 2020

Table 10: Effect on lymphocyte count

\begin{tabular}{|c|c|c|c|c|c|c|}
\hline DC (L) & Mean & n & SD & SE & T value & P value \\
\hline Pre & 33.44 & \multirow{2}{*}{30} & 3.72897 & 0.68081 & -1.429 & 0.164 \\
& & & & & \\
Post & 34.03 & & 3.74802 & 0.68429 & & \\
\hline Post & 34.03 & \multirow{2}{*}{30} & 3.74802 & 0.68429 & 0.840 & 0.408 \\
Follow up & 33.53 & & 3.83822 & 0.70076 & & \\
\hline Pre & 33.44 & \multirow{2}{*}{30} & 3.72897 & 0.68081 & -0.144 & 0.886 \\
Follow up & 33.53 & & 3.83822 & 0.70076 & & \\
\hline
\end{tabular}

SD - standard deviation; SE - standard error; significant when $\mathrm{p} \leq 0.05$

Table 11: Effect on neutrophil count

\begin{tabular}{|c|c|c|c|c|c|c|}
\hline $\mathrm{DC}(\mathrm{N})$ & Mean & $\mathbf{n}$ & SD & SE & T value & $P$ value \\
\hline $\begin{array}{c}\text { Pre } \\
\text { Post }\end{array}$ & $\begin{array}{r}57.7 \\
59.09\end{array}$ & 30 & $\begin{array}{r}3.633 \\
4.1066\end{array}$ & $\begin{array}{l}0.6633 \\
0.7497\end{array}$ & 2.953 & 0.0062 \\
\hline $\begin{array}{c}\text { Post } \\
\text { Follow up }\end{array}$ & $\begin{array}{r}59.09 \\
58.836 \\
\end{array}$ & 30 & $\begin{array}{r}.1066 \\
4.312 \\
\end{array}$ & $\begin{array}{r}0.7497 \\
0.787 \\
\end{array}$ & 0.513 & 0.612 \\
\hline $\begin{array}{c}\text { Pre } \\
\text { Follow up } \\
\end{array}$ & $\begin{array}{r}57.7 \\
58.836 \\
\end{array}$ & 30 & $\begin{array}{l}3.633 \\
4.312 \\
\end{array}$ & $\begin{array}{r}0.6633 \\
0.787 \\
\end{array}$ & 2.793 & 0.009 \\
\hline
\end{tabular}

$\mathrm{SD}$ - standard deviation; SE - standard error; significant when $\mathrm{p} \leq 0.05$

Table 12: Effect on mid cell count

\begin{tabular}{|c|c|c|c|c|c|c|}
\hline DC (mid) & Mean & n & SD & SE & T value & P value \\
\hline Pre & 8.86 & \multirow{2}{*}{30} & 1.3637 & 0.248 & 2.854 & 0.008 \\
Post & 7.796 & & 1.794 & 0.327 & & \\
\hline Post & 7.796 & \multirow{2}{*}{30} & 1.794 & 0.327 & 0.008 & 0.9935 \\
Follow up & 7.8 & & 2.097 & 0.383 & & \\
\hline Pre & 8.86 & 30 & 1.3637 & 0.248 & 2.549 & 0.016 \\
Follow up & 7.8 & & 2.0965 & 0.383 & & \\
\hline
\end{tabular}

$\mathrm{SD}$ - standard deviation; SE - standard error; significant when $\mathrm{p} \leq 0.05$

Table 13: Effect on ESR in males

\begin{tabular}{|c|c|c|c|c|c|c|}
\hline ESR (males) & Mean & $\mathbf{n}$ & SD & SE & T value & P value \\
\hline Pre & 21.5 & 30 & 7.717 & 2.228 & 0.710 & 0.493 \\
Post & 20.5 & & 5.776 & 1.667 & & \\
\hline Post & 20.5 & 30 & 5.776 & 1.667 & -0.364 & 0.723 \\
Follow up & 20.75 & & 5.61 & 1.619 & & \\
\hline Pre & 21.5 & 30 & 7.717 & 2.228 & 0.631 & 0.541 \\
Follow up & 20.75 & & 5.61 & 1.619 & & \\
\hline
\end{tabular}

$\mathrm{SD}$ - standard deviation; SE - standard error; significant when $\mathrm{p} \leq 0.05$

Table 14: Effect on ESR in females

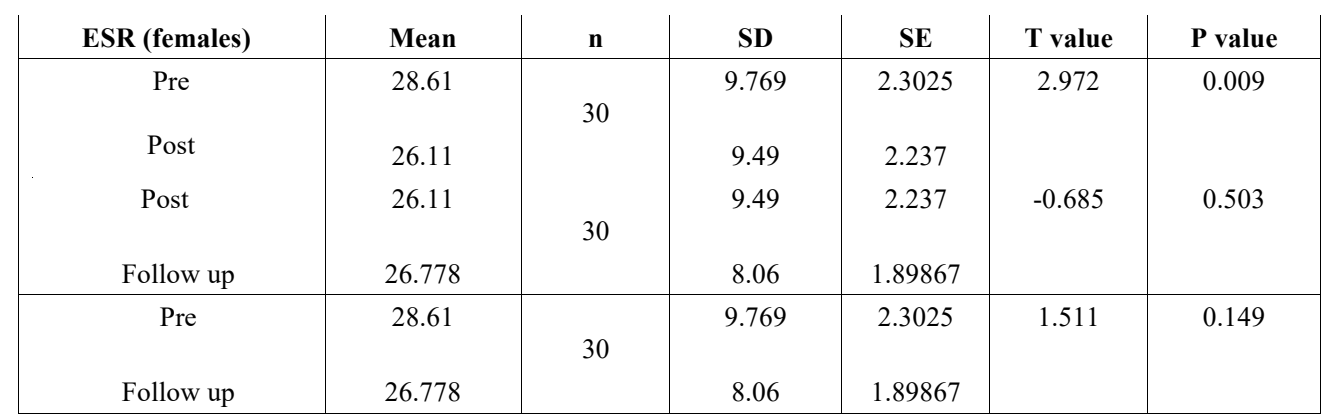

$\mathrm{SD}$ - standard deviation; SE - standard error; significant when $\mathrm{p} \leq 0.05$ 
Jaseena C. et al / Int. J. Res. Ayurveda Pharm. 11 (4), 2020

Table 15: Effect on Total Cholesterol

\begin{tabular}{|c|c|c|c|c|c|c|}
\hline Total cholesterol & Mean & $\mathbf{n}$ & SD & SE & T value & P value \\
\hline Pre & 189.961 & \multirow{2}{*}{30} & 11.974 & 2.186 & 3.66 & 0.001 \\
\hline Post & 186.329 & & 13.205 & 2.4108 & & \\
\hline Post & 186.329 & \multirow{2}{*}{30} & 13.205 & 2.4108 & 1.347 & 0.189 \\
\hline Follow up & 184.285 & & 16.473 & 3.007 & & \\
\hline Pre & 189.961 & \multirow{2}{*}{30} & 11.974 & 2.186 & 3.387 & 0.002 \\
\hline Follow up & 184.285 & & 16.473 & 3.007 & & \\
\hline
\end{tabular}

$\mathrm{SD}$ - standard deviation; SE - standard error; significant when $\mathrm{p} \leq 0.05$

Table 16: Effect on HDL

\begin{tabular}{|c|c|c|c|c|c|c|}
\hline HDL & Mean & n & SD & SE & T value & P value \\
\hline Pre & 51.597 & \multirow{2}{*}{30} & 6.321 & 1.154 & 2.75 & 0.01 \\
Post & 54.65 & & 6.066 & 1.107 & & 0.11 \\
\hline Post & 54.65 & 30 & 6.066 & 1.107 & 1.653 & \\
Follow up & 53.637 & & 5.188 & 0.947 & & 0.015 \\
\hline Pre & 51.597 & 30 & 6.321 & 1.154 & 2.592 & \\
Follow up & 53.637 & & & & & \\
\hline
\end{tabular}

SD - standard deviation; SE - standard error; significant when $\mathrm{p} \leq 0.05$

Table 17: Effect on LDL

\begin{tabular}{|c|c|c|c|c|c|c|}
\hline LDL & Mean & n & SD & SE & T value & $P$ value \\
\hline $\begin{array}{c}\text { Pre } \\
\text { Post }\end{array}$ & $\begin{array}{c}109.659 \\
104.45\end{array}$ & 30 & $\begin{array}{r}14.36 \\
15.746\end{array}$ & $\begin{array}{l}2.62 \\
2.87\end{array}$ & 3.972 & 0.000 \\
\hline $\begin{array}{c}\text { Post } \\
\text { Follow up }\end{array}$ & $\begin{array}{l}104.45 \\
104.26\end{array}$ & 30 & $\begin{array}{r}15.746 \\
16.45 \\
\end{array}$ & $\begin{array}{r}2.87 \\
3.004 \\
\end{array}$ & 0.107 & 0.916 \\
\hline $\begin{array}{c}\text { Pre } \\
\text { Follow up }\end{array}$ & $\begin{array}{l}109.659 \\
104.26\end{array}$ & 30 & $\begin{array}{l}14.36 \\
16.45 \\
\end{array}$ & $\begin{array}{r}2.62 \\
3.004 \\
\end{array}$ & 2.789 & 0.009 \\
\hline
\end{tabular}

SD - standard deviation; SE - standard error; significant when $\mathrm{p} \leq 0.05$

Table 18: Effect on VLDL

\begin{tabular}{|c|c|c|c|c|c|c|}
\hline VLDL & Mean & n & SD & SE & T value & P value \\
\hline $\begin{array}{c}\text { Pre } \\
\text { Post }\end{array}$ & $\begin{array}{l}27.6585 \\
26.9047\end{array}$ & 30 & $\begin{array}{l}3.30266 \\
3.34841\end{array}$ & $\begin{array}{l}0.60298 \\
0.61133\end{array}$ & 2.812 & 0.009 \\
\hline $\begin{array}{c}\text { Post } \\
\text { Follow up }\end{array}$ & $\begin{array}{l}26.9047 \\
26.7627\end{array}$ & 30 & $\begin{array}{l}3.34841 \\
2.53557\end{array}$ & $\begin{array}{l}0.61133 \\
0.46293\end{array}$ & 0.449 & 0.657 \\
\hline $\begin{array}{c}\text { Pre } \\
\text { Follow up }\end{array}$ & $\begin{array}{l}27.6585 \\
26.7627\end{array}$ & 30 & $\begin{array}{l}3.30266 \\
2.53557\end{array}$ & $\begin{array}{l}0.60298 \\
0.46293\end{array}$ & 2.311 & 0.028 \\
\hline
\end{tabular}

$\mathrm{SD}$ - standard deviation; SE - standard error; significant when $\mathrm{p} \leq 0.05$

Table 19: Effect on triglycerides

\begin{tabular}{|c|c|c|c|c|c|c|}
\hline Triglycerides & Mean & n & SD & SE & T value & P value \\
\hline Pre & 143.348 & \multirow{2}{*}{30} & 13.88 & 2.534 & 3.613 & 0.001 \\
Post & 136.99 & & 14.636 & 2.672 & & 0.469 \\
\hline Post & 136.99 & \multirow{2}{*}{30} & 14.636 & 2.672 & 0.734 & \\
\hline Follow up & 136.0003 & & 16.120 & 2.943 & & 0.0037 \\
\hline Pre & 143.348 & \multirow{2}{*}{30} & 13.88 & 2.534 & 3.1593 & \\
\hline
\end{tabular}

SD - standard deviation; SE - standard error; significant when $\mathrm{p} \leq 0.05$ 
Table 20: Effect on Medo dhatu

\begin{tabular}{|c|c|c|c|c|c|c|}
\hline Medo dhatu & Mean & n & SD & SE & T value & P value \\
\hline Pre & 44.466 & \multirow{2}{*}{30} & 3.14844 & 0.57482 & 3.693 & 0.001 \\
Post & 41.466 & & 3.94561 & 0.72037 & & \\
\hline Post & 41.466 & 30 & 3.94561 & 0.72037 & -0.379 & 0.707 \\
Follow up & 41.766 & & 3.39049 & 0.61902 & & \\
\hline Pre & 44.466 & 30 & 3.14844 & 0.57482 & 3.036 & 0.005 \\
Follow up & 41.766 & 3.39049 & 0.61902 & & \\
\hline
\end{tabular}

Table 21: Effect on temperature

\begin{tabular}{|c|c|c|c|c|c|c|}
\hline Temperature & Mean & n & SD & SE & T value & P value \\
\hline Pre & 98.5100 & \multirow{2}{*}{30} & 0.45830 & 0.08367 & -0.0463 & 0.963 \\
& & & & & \\
Post & 98.5114 & & 0.34867 & 0.06366 & & \\
\hline
\end{tabular}

$\mathrm{SD}$ - standard deviation; SE - standard error; significant when $\mathrm{p} \leq 0.05$

Table 22: Effect on systolic BP

\begin{tabular}{|c|c|c|c|c|c|c|}
\hline Systolic BP & Mean & n & SD & SE & T value & P value \\
\hline Pre & 129.74 & \multirow{2}{*}{30} & 12.09355 & 2.20797 & -0.917 & 0.367 \\
Post & 130.33 & & 12.02339 & 2.19516 & & \\
\hline
\end{tabular}

SD - standard deviation; SE - standard error; significant when $\mathrm{p} \leq 0.05$

Table 23: Effect on diastolic BP

\begin{tabular}{|c|c|c|c|c|c|c|}
\hline Diastolic BP & Mean & N & SD & SE & T value & P value \\
\hline Pre & 86.0571 & \multirow{2}{*}{30} & 9.95596 & 1.81770 & 0.261 & 0.796 \\
& & & 10.11409 & 1.84657 & & \\
Post & 86.0064 & & & & \\
\hline
\end{tabular}

SD - standard deviation; SE - standard error; significant when $\mathrm{p} \leq 0.05$

Table 24: Effect on pulse

\begin{tabular}{|c|c|c|c|c|c|c|}
\hline Pulse & Mean & n & SD & SE & T value & P value \\
\hline Pre & 76.6286 & \multirow{2}{*}{30} & 6.92125 & 1.26364 & 0.832 & 0.412 \\
& & & & & \\
Post & 76.4466 & & 7.31841 & 1.33615 & & \\
\hline
\end{tabular}

SD - standard deviation; SE - standard error; significant when $\mathrm{p} \leq 0.05$

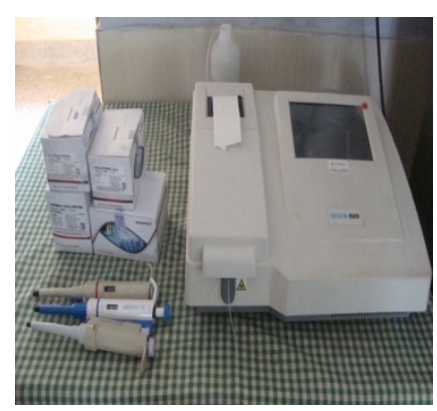

Figure 1: Semi auto analyser

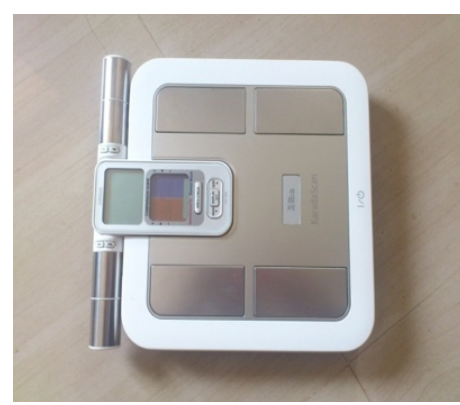

Figure 2: Body composition monitor

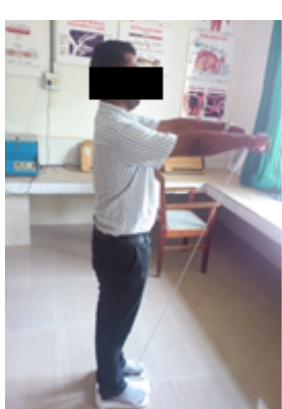

Figure 3: Assessment of body parameters using body composition monitor

\section{RESULTS AND DISCUSSION}

$40 \%$ of participants were belonging to the age group of $30-35$ years and rest $60 \%$ of participants were from 36-40 years age group. $40 \%$ of participants were males and rest $60 \%$ of participants were females. Participants belonging to Hindu religion formed $56.66 \%$ of the total participants. $40 \%$ participants were belonging to Muslim community. The remaining 3.33\% participants were from Christian religion. $46.66 \%$ of participants were having school level education and $53.33 \%$ of participants were graduates. $33.33 \%$ of participants were unemployed.
$26.66 \%$ of participants were having government job. $26.66 \%$ of participants were having job in private sector and $13.33 \%$ of participants were doing cooli. All the participants in this study were belonging to the rural area. $16.66 \%$ of participants were poor. $43.33 \%$ of participants were belonging to middle class and $40 \%$ were belonging to upper class. $76.66 \%$ of participants were having normal bowel and the remaining $23.33 \%$ were having hard stool. $83.33 \%$ of participants were taking mixed diet and rest $16.66 \%$ participants were taking vegetarian diet. $26.66 \%$ of participants were taking less amount of food, $43.33 \%$ of participants were taking moderate amount of food and the rest 
$30 \%$ were taking more food. $60 \%$ of participants were having sedentary work. $26.66 \%$ of participants were having household activity and remaining $13.33 \%$ were having hard work. $40 \%$ of participants were having Pravara Ahara Shakti. 40\% of participants were having Madhyama Ahara Shakti and the remaining 20\% were having Avara Ahara Shakti.

The mean value of body weight before trial was 73.778 with SD 10.27545. After trial it was 72.9570 and 10.17951 respectively, with a $P$ value of 0.001 which was statistically significant. After follow up the mean value were 73.351 with SD 10.36050. On comparison with the values of post and follow up assessment, the observed $P$ value was 0.109 which was statistically insignificant. On comparison with the values of pre and follow up assessment, the observed $P$ value was 0.009 which was statistically significant (Table 2).

The mean value of BMI before trial was 27.143 with SD 1.455. After trial it was 26.9133 and 1.5131 respectively, with a $P$ value of 0.017 which was statistically significant. After follow up the mean value were 27.0167 with SD 1.47603. On comparison with the values of post and follow up assessment, the observed $P$ value was 0.227 which was statistically insignificant. On comparison with the values of pre and follow up assessment, the observed $\mathrm{P}$ value was 0.045 which was statistically significant (Table 3 ).

The mean value of body fat percentage before trial was 37.5667 with SD 5.83363. After trial it was 37.02 and 5.84940 respectively, with a $\mathrm{P}$ value of 0.001 which was statistically significant. After follow up the mean value were 37.21 with SD 5.66473. On comparison with the values of post and follow up assessment, the observed $\mathrm{P}$ value was 0.352 which was statistically insignificant. On comparison with the values of pre and follow up assessment, the observed $\mathrm{P}$ value was 0.01 which was statistically significant (Table 4 ).

The mean value of skeletal muscle percentage before procedure was 25.05 with SD 4.06191. After trial it was 24.83 and 4.0875 respectively, with a $\mathrm{P}$ value of 0.009 which was statistically significant. After follow up the mean value were 24.07 with SD 4.20797. On comparison with the values of post and follow up assessment, the observed $P$ value was 0.05 which was statistically insignificant. On comparison with the values of pre and follow up assessment, the observed $P$ value was 0.016 which was statistically significant (Table 5).

The mean value of visceral fat level before procedure was 14.733 with SD 4.98918. After trial it was 14.433 and 4.96667 respectively, with a $\mathrm{P}$ value of 0.005 which was statistically significant. After follow up the mean value were 14.5667 with SD of 4.96667. On comparison with the values of post and follow up assessment, the observed $\mathrm{P}$ value was 0.293 which was statistically insignificant. On comparison with the values of pre and follow up assessment, the observed $P$ value was 0.134 which was statistically insignificant (Table 6).

The mean value of $\mathrm{Hb}$ in males before trial was 12.93 with SD 1.033. After trial it was 13.28 and 1.008 respectively, with a $P$ value of 0.012 which was statistically significant. After follow up the mean value were 13.13 with SD 1.026. On comparison with the values of post and follow up assessment, the observed $P$ value was 0.390 which was statistically insignificant. On comparison with the values of pre and follow up assessment, the observed $\mathrm{P}$ value was 0.379 which was statistically insignificant (Table 7).

The mean value of $\mathrm{Hb}$ in females before trial was 12.45 with SD 1.125. After trial it was 12.7 and 1.105 respectively, with a $P$ value of 0.098 which was statistically insignificant. After follow up the mean value were 12.59 with SD 1.234. On comparison with the values of post and follow up assessment, the observed $P$ value was 0.616 which was statistically insignificant. On comparison with the values of pre and follow up assessment, the observed $P$ value was 0.228 which was statistically insignificant (Table 8).

The mean value of TLC before trial was $8.2733 \times 10^{3}$ with SD $1.69032 \times 10^{3}$. After trial it was $7.7167 \times 10^{3}$ and $1.63371 \times 10^{3}$ respectively, with a $\mathrm{P}$ value of 0.003 which was statistically significant. After follow up the mean value was $7.8867 \times 10^{3}$ with SD $1.54601 \times 10^{3}$. On comparison with the values of post and follow up assessment, the observed $P$ value was 0.259 which was statistically insignificant. On comparison with the values of pre and follow up assessment, the observed $\mathrm{P}$ value was 0.024 which was statistically significant (Table 9).

The mean value of lymphocyte count before trail was 33.44 with SD 3.72897. After trial it was 34.03 and 3.74802 respectively, with a $\mathrm{P}$ value of 0.164 which was statistically insignificant. After follow up the mean value were 33.53 with SD of 3.83822 . On comparison with the values of post and follow up assessment, the observed $\mathrm{P}$ value was 0.408 which was statistically insignificant. On comparison with the values of pre and follow up assessment, the observed $\mathrm{P}$ value was 0.886 which was statistically insignificant (Table 10).

The mean value of neutrophil count before trial was 57.7 with SD of 3.633. After trial it was 59.09 and 4.1066 respectively, with a $P$ value of 0.0062 which was statistically significant. After follow up the mean value were 58.836 with SD of 4.312. On comparison with the values of post and follow up assessment, the observed $\mathrm{P}$ value was 0.612 which was statistically insignificant. On comparison with the values of pre and follow up assessment, the observed $\mathrm{P}$ value was 0.009 which was statistically significant (Table 11).

The mean value of mid cell count before trial was 8.86 with SD of 1.3637. After trial it was 7.796 and 1.794 respectively with a $P$ value of 0.008 which was statistically significant. After follow up the mean value were 7.8 with SD 2.097. On comparison with the values of post and follow up assessment, the observed $\mathrm{P}$ value was 0.9935 which was statistically insignificant. On comparison with the values of pre and follow up assessment, the observed $\mathrm{P}$ value was 0.016 which was statistically significant (Table 12).

The mean value of ESR in males before trial was 21.5 with SD 7.717. After trial it was 20.5 and 5.776 respectively, with a $P$ value of 0.493 which was statistically insignificant. After follow up the mean value were 20.75 with SD 5.61. On comparison with the values of post and follow up assessment, the observed $P$ value was 0.723 which was statistically insignificant. On comparison with the values of pre and follow up assessment, the observed $\mathrm{P}$ value was 0.541 which was statistically insignificant (Table 13).

The mean value of ESR in females before trial was 28.61 with SD 9.769. After trial it was 26.11 and 9.49 respectively, with a $P$ value of 0.009 which was statistically significant. After follow up the mean value were 26.778 with SD 8.06. On comparison with the values of post and follow up assessment, the observed $P$ value was 0.503 which was statistically insignificant. On comparison with the values of pre and follow up assessment, the observed $\mathrm{P}$ value was 0.149 which was statistically insignificant (Table 14).

The mean value of Total Cholesterol before trial was 189.961 with SD 11.974. After trial it was 186.329 and 13.205 respectively, with a $\mathrm{P}$ value of 0.001 which was statistically significant. After follow up the mean value were 184.285 with SD 
16.473. On comparison with the values of post and follow up assessment, the observed $\mathrm{P}$ value was 0.189 which was statistically insignificant. On comparison with the values of pre and follow up assessment, the observed $P$ value was 0.002 which was statistically significant (Table 15).

The mean value of HDL before trial was 51.597 with SD 6.321 . After trial it was 54.65 and 6.066 respectively, with a $P$ value of 0.01 which was statistically significant. After follow up the mean value were 53.637 with SD 5.188. On comparison with the values of post and follow up assessment, the observed $\mathrm{P}$ value was 0.11 which was statistically insignificant. On comparison with the values of pre and follow up assessment, the observed $P$ value was 0.015 which was statistically significant (Table 16).

The mean value of LDL before trial was 109.659 with SD 14.36. After trial it was 104.45 and 15.746 respectively, with a $P$ value of 0.000 which was statistically significant. After follow up the mean value were 104.26 with SD 16.45. On comparison with the values of post and follow up assessment, the observed $\mathrm{P}$ value was 0.916 which was statistically insignificant. On comparison with the values of pre and follow up assessment, the observed $\mathrm{P}$ value was 0.009 which was statistically significant (Table 17).

The mean value of VLDL before trial was 27.6585 with SD 3.30266. After trial it was 26.9047 and 3.34841 respectively, with a $P$ value 0.009 which was statistically significant. After follow up the mean value were 26.7627 with SD 2.53557. On comparison with the values of post and follow up assessment, the observed $P$ value was 0.657 which was statistically insignificant. On comparison with the values of pre and follow up assessment, the observed $\mathrm{P}$ value was 0.028 which was statistically significant (Table 18).

The mean value of triglycerides before trial was 143.348 with SD 13.88. After trial it was 136.99 and 14.636 respectively, with a $P$ value 0.001 which was statistically significant. After follow up the mean value were 136.0003 with SD 16.120. On comparison with the values of post and follow up assessment, the observed $\mathrm{P}$ value was 0.469 which was statistically insignificant. On comparison with the values of pre and follow up assessment, the observed $\mathrm{P}$ value was 0.0037 which was statistically significant (Table 19).

The mean value of the vitiation of medo dhatu before trial was 44.466 with SD 3.14844. After trial it was 41.466 and 3.94561 respectively, with a $\mathrm{P}$ value 0.001 which was statistically significant. After follow up the mean value were 41.766 with SD 3.39049. On comparison with the values of post and follow up assessment, the observed $\mathrm{P}$ value was 0.707 which was statistically insignificant. On comparison with the values of pre and follow up assessment, the observed $P$ value was 0.005 which was statistically significant (Table 20).

The mean value of temperature before trial was 98.51 with SD 0.45830 . After trial it was 98.5114 and 0.34867 respectively, with a $P$ value 0.963 which was statistically insignificant (Table 21 ).

The mean value of systolic BP before trial was 129.74 with SD 12.09355. After trial it was 130.33 and 12.02339 respectively, with a $\mathrm{P}$ value 0.367 which was statistically insignificant(Table 22).

The mean value of diastolic BP before trial was 86.0571 with SD 9.95596. After trial it was 86.0064 and 10.11409 respectively, with a $P$ value 0.796 which was statistically insignificant (Table 23).
The mean value of pulse before trial was 76.6286 with SD 6.92125. After trial it was 76.4466 and 7.31841 respectively, with $P$ value 0.412 which was statistically insignificant (Table 24).

Udvartana alleviates pain by neural gating mechanism and by reducing the sensitivity of pain receptors. It helps to get rid of body odour by removing the bacteria and the substances, the conversion of which cause body odour. By siramukha vishodhana guna, udvartana reduces itching. It decreases tandra by improving systemic circulation especially cerebral circulation. Due to the medo hara property of udvartana, it reduces cholesterol and thereby hinders the testosterone binding by the cholesterol. This action helps to increase the availability of testosterone. Bhrajaka pitta is the metabolic epithelial enzymes which cause the deepana and pachana of substances applied on twak in processes like abhyanga, udvartana etc. Udvartana causes vasodilatation which helps to improve the skin's colour. With the help of Bhrajaka pitta, laghu and ruksha properties of triphala reduce kapha. Shrotorodha in sthaulya is comparable to leptin receptor resistance. The range of substances that come under the concept of medo dhatu comprises all lipid substances present in the body i.e., lipid bilayer of the cell, triglycerides, cholesterol, phospholipids, fatty acids etc.

\section{CONCLUSION}

It can be concluded that udvartana can be effectively carried out to reduce the medo dhatu significantly using body composition monitor.

\section{REFERENCES}

1. Bhurosy T, Jeewon R. Overweight and Obesity Epidemic in Developing Countries: A Problem with Diet, Physical Activity, or Socioeconomic Status? Sci World J 2014; 2014: 964236.

2. Stevens GA, Singh GM, Lu Y, Danaei G, Lin JK, Finucane $\mathrm{MM}$, et al. National, regional and global trends in adult overweight and obesity prevalence. Popul Health Metr 2012; 10(1): 22.

3. Alex Vass. Obesity causes 30000 deaths a year, report says. BMJ 2002; 324(7331): 192.

4. Hurt RT, Kulisek C, Buchanan LA, Mc Clave SA. The Obesity Epidemic: Challenges, Health Initiatives and Implications for Gastroenterologists. Gastroenterol Hepatol (N Y) 2010; 6(12): 780-792.

5. Kokiwar PR. An epidemiological study of obesity in a rural area. J Family Community Med 2011; 18(2): 91.

6. Reddy KS, Prabhakaran D, Shah P, Shah B. Differences in body mass index and waist: hip ratios in North Indian rural and urban populations. Obes Rev 2002; 3: 197-202.

7. Abdelaal M, le Roux CW, Docherty NG. Morbidity and mortality associated with obesity. Ann Transl Med 2017; 5(7): 161.

8. Wells JCK, Fewtrell MS. Measuring body composition. Arch Dis Child 2006; 91(7): 612-617.

9. Nuttall FQ. Body Mass Index - Obesity, BMI, and Health: A Critical Review. Nutr Today 2015; 50(3): 117-128.

10. Gallagher D, Heymsfield SB, Heo M, Jebb SA, Murgatroyd PR, Sakamoto Y. Healthy percentage body fat ranges: an approach for developing guidelines based on body mass index. Am J Clin Nutr 2000; 72(3): 694-701.

11. Kurniawan LB, Bahrun U, Hatta M, Arif M. Body Mass, Total Body Fat Percentage, and Visceral Fat Level Predict Insulin Resistance Better Than Waist Circumference and Body Mass Index in Healthy Young Male Adults in Indonesia. J Clin Med 2018; 7(5): 96. 
12. Peterson CT, Denniston BSK, Chopra D. Therapeutic Uses of triphala in Ayurvedic Medicine. J Altern Complement Med 2017; 23(8): 607-614.

\section{Cite this article as:}

Jaseena C et al. Evaluation of effect of Udvartana on Medo dhatu using Body Composition Monitor. Int. J. Res. Ayurveda Pharm. 2020;11(4):59-69 http://dx.doi.org/10.7897/2277-4343.110490

\section{Source of support: Nil, Conflict of interest: None Declared}

Disclaimer: IJRAP is solely owned by Moksha Publishing House - A non-profit publishing house, dedicated to publishing quality research, while every effort has been taken to verify the accuracy of the content published in our Journal. IJRAP cannot accept any responsibility or liability for the site content and articles published. The views expressed in articles by our contributing authors are not necessarily those of IJRAP editor or editorial board members. 